\title{
Meditação, Bem-Estar e a Ciência Psicológica: Revisão de Estudos Empíricos
}

\author{
Carolina Baptista Menezes", Débora Dalbosco Dell'Aglio \& Lisiane Bizarro \\ Universidade Federal do Rio Grande do Sul, Porto Alegre, Brasil
}

\begin{abstract}
RESUMO
A meditação sentada e silenciosa pode ser descrita como um treinamento mental, o qual tem sido tradicionalmente associado ao bem-estar. $\mathrm{O}$ crescente interesse científico pela prática tem ajudado a demonstrar esta relação através de diferentes metodologias. Este trabalho objetiva discutir a interface entre alguns pressupostos da meditação e da ciência psicológica no que tange ao bem-estar, assim como resultados empíricos que parecem apoiar o efeito da prática sobre experiências emocionais positivas. $\mathrm{O}$ artigo também propõe que esta relação ocorre mediada por três processos psicológicos principais e interdependentes: relaxamento, metacognição/mindfulness e autorregulação. Discute-se que a investigação destes, assim como dos efeitos da meditação em geral, pode auxiliar na sua aplicação no campo da psicologia, assim como no desenvolvimento da ciência psicológica.
\end{abstract}

Palavras-chave: meditação; bem-estar; relaxamento; mindfulness; autorregulação.

\section{ABSTRACT \\ Meditation, Well-Being and Psychological Science: A Review of Empirical Studies}

Sitting and silent meditation can be described as a mental training, which has been traditionally associated with well-being. The growing scientific interest in it has helped confirm this association through various methodologies. This work aims to discuss the interface between some of the tenets of meditation and psychological science concerning well-being, as well as empirical results supporting the effects of this practice on positive emotional experiences. It also proposes that this relationship is mediated by three main and interdependent psychological processes: relaxation, metacognition/ mindfulness, and self-regulation. We discuss that the investigation of such mechanisms and the general effects of meditation can contribute to its application in the field of psychology, as well as to the development of the psychological science.

Keywords: meditation; well-being; relaxation; mindfulness; self-regulation.

\section{MEDITAÇÃO - O QUE É?}

Meditação é um termo amplo, que pode englobar definições distintas conforme o contexto filosóficoreligioso no qual a prática está inserida (Walsh \& Shapiro, 2006). Neste artigo serão abordados alguns achados encontrados nas últimas décadas sobre a meditação passiva, ou meditação sentada e silenciosa, como também é conhecida. Este é o tipo de meditação que mais vem sendo pesquisado cientificamente, tanto em sua forma original, oriunda das tradições espirituais do oriente, como na sua forma secular, adaptada ao ocidente. Ambas utilizam métodos muito semelhantes e têm como objetivo a busca pelo funcionamento psi- cológico saudável. A diferença é que na meditação oriental a aspiração ao desenvolvimento pessoal está intrinsecamente relacionada à dimensão espiritual, a qual não é necessariamente enfatizada e/ou vinculada à meditação secular (Walsh \& Shapiro, 2006).

Diferentemente da meditação ativa - com movimento - e das meditações em que se pensa sobre um tema, como a analítica e a cristã, por exemplo, a meditação sentada e silenciosa tem sido descrita como uma forma de treinamento mental que consiste na focalização prolongada e consciente da atenção sobre um único objeto, tal como a respiração, ou sobre a própria consciência (Lutz, Dunne, \& Davidson, 2007). O ob-

\footnotetext{
* Endereço para correspondência: Carolina Baptista Menezes: menezescarolina@ hotmail.com
} 
jetivo é aprender a não elaborar e desvincular-se de distrações e pensamentos discursivos e/ou ruminativos. Alguns autores descrevem a aquisição de tal habilidade - focar a atenção na consciência do momento presente, sem a elaboração dos conteúdos mentais como um estado de liberdade psicológica (Martin, 1997) ou um estado de liberdade da poluição mental (Austin, 1998).

Um dos princípios da meditação é que o treino da concentração leva à superação da distração, de forma que quanto mais concentrada a mente estiver, menos perturbação ela sofrerá. Consequentemente, quanto menos a mente se perturbar, menos ela estará suscetível a desequilíbrios ou, dito de outra forma, quanto mais concentrada a mente estiver, tão mais estável ela estará. É por isso que, tradicionalmente, diz-se que o objetivo da meditação é facilitar e gerar uma estabilidade mental e emocional (Wallace \& Shapiro, 2006).

Operacionalmente, a meditação sentada e silenciosa tem sido descrita a partir de um conjunto de cinco parâmetros: 1) deve ser uma técnica claramente definida, 2) com a qual se alcança algum relaxamento físico e 3) da lógica ao longo do processo, 4) sendo um estado autoinduzido, 5) através da utilização de um foco (Cardoso, Souza, Camano, \& Leite, 2004). O foco, ou âncora, pode ser fechado, caracterizando o subtipo meditativo concentrativo, também chamado de meditação da atenção focada, ou pode ser um foco aberto, caracterizando o subtipo meditativo mindfulness, também designado de meditação do monitoramento aberto (Davidson \& Lutz, 2008).

Através das características citadas até aqui, podese perceber aspectos comuns entre esta prática e a ciência psicológica. Da mesma forma que nas psicoterapias, por exemplo, através da meditação também se busca a redução do sofrimento psicológico e o cultivo de qualidades positivas, como o bem-estar. Este seria um dos principais pontos convergentes entre as práticas meditativas e a psicologia, pois ambas se apóiam na premissa de que é possível conquistar níveis cada vez maiores de realização pessoal e que este processo depende do próprio paciente ou do próprio praticante, através da auto-observação e do crescente conhecimento de si (Martin, 1997; Wallace \& Shapiro, 2006).

No que se refere à meditação, esta é comumente e popularmente associada ao bem-estar, sendo este um dos principais apelos para a sua prática. Uma das contribuições das pesquisas nesta área tem sido mostrar alguns resultados convergentes sugerindo a sua asso- ciação a desfechos psicológicos positivos. O presente trabalho pretende fazer uma revisão teórica, apresentando e discutindo a interface entre alguns pressupostos da meditação e da ciência psicológica, assim como apresentar uma revisão da literatura que investigou três processos psicológicos associados à prática da meditação: relaxamento, metacognição/mindfulness e autorregulação. Isso porque o argumento central neste artigo é a proposição de que estes parecem ser mediadores fundamentais da relação entre meditação e bemestar. Com isso, buscou-se destacar a relevância do estudo científico da meditação, especialmente no campo da psicologia, concluindo com uma observação sobre o estado da área no Brasil.

Ressalta-se que alguns autores argumentam que as diferentes práticas de meditação devem ser estudadas separadamente, visto que em uma perspectiva neurocientífica, elas se caracterizam por circuitos neurais distintos, que por sua vez produzem efeitos particulares. Essa distinção não se aplica apenas à meditação passiva e ativa, mas também às modalidades do tipo passivo, tal como o foco fechado - concentrativa - e aberto - mindfulness (Lutz e cols., 2007). Estas diferenças não serão priorizadas neste artigo. De um ponto de vista fenomenológico, entende-se que a experiência e o objetivo final da meditação é a busca por um estado de maior equilíbrio e bem-estar psicológico, de forma que os resultados apresentados referem-se à meditação de foco fechado e aberto.

\section{MÉTODO}

Este artigo incluiu a busca de artigos sem limite de data, através dos descritores "meditação", "mindfulness", "relaxamento", "metacognição", "autorregulção", "bem-estar" e "emoções positivas". Foram acessadas as bases de dados Psychinfo, Pubmed, Scielo e PEPsic. Utilizou-se diferentes combinações dos descritores, a fim de abranger o maior número de estudos com essas temáticas. Os artigos incluídos compreenderam o período entre 1976 e 2010, sendo a maioria da última década. Foram excluídos estudos que não tratavam da meditação sentada e silenciosa e cuja amostra não era de adultos. Tendo em vista o objetivo do presente trabalho, ressalta-se que esta não foi uma revisão sistemática, e sim uma revisão teórica, em que os artigos foram buscados, apresentados e discutidos de acordo com as categorias estipuladas a priori: relaxamento, metacognição/mindfulness e autorregulção. 


\section{MEDITAÇÃO E BEM-ESTAR}

Existem diferentes formas de se medir e se referir ao bem-estar na literatura científica, tais como felicidade, qualidade de vida, bem-estar subjetivo, bemestar psicológico, satisfação de vida, entre outros. De forma abrangente, o bem-estar pode ser definido como um ótimo funcionamento psicológico (Veenhoven, 1997). Na perspectiva do conceito de qualidade de vida, este funcionamento será ótimo na medida em que o sujeito avaliar os diferentes âmbitos da sua vida - físico, psicológico, nível de independência, relações sociais, ambiente e espiritual - como satisfatórios (Fleck e cols., 1999). Na perspectiva do conceito de bem-estar psicológico, proposto por Ryff e Keyes (1995), o qual deu origem à Escala de Bem-Estar Psicológico - PWBS, o funcionamento positivo ou a felicidade resultam do equilíbrio dos seguintes domínios: autoaceitação, crescimento pessoal, objetivo na vida, relações positivas com os outros, domínio do meio e autonomia. Já segundo a perspectiva do construto de bem-estar subjetivo, conforme proposto por Diener, Lucas e Oishi (2005), o funcionamento psicológico positivo resulta da avaliação que o sujeito faz acerca de sua vida, a qual depende de aspectos afetivos e cognitivos, os quais são comumente investigados através da Escala de Afetos Positivos e Afetos Negativos - PANAS (Diener e cols., 2005). Os aspectos afetivos correspondem às experiências emocionais, representadas pela preponderância dos afetos positivos sobre os negativos. A avaliação cognitiva baseia-se no julgamento da satisfação de vida como um todo, perpassando os âmbitos do trabalho, da família, do lazer, da saúde, das finanças, do selfe do grupo.

Estas perspectivas fornecem uma base inicial sobre a qual é possível traçar alguns paralelos existentes entre meditação e bem-estar. Em termos conceituais, por exemplo, a importância de como as pessoas avaliam suas experiências para o seu bem-estar (Diener e cols., 2005; Fleck e cols., 1999) é um ponto comum fundamental com a prática meditativa. Isto porque através da meditação busca-se mudar a consciência com a qual se percebe as experiências, ao invés de mudar as experiências em si (Bishop e cols., 2004). Portanto, ao conseguir mudar e controlar a percepção das coisas, o praticante pode fazer com que essa percepção favoreça o seu bem-estar.

Esta ênfase na forma com que se percebe o mundo e a si mesmo também caracteriza uma importante semelhança entre os pressupostos da prática meditativa e da psicoterapia cognitiva (Walsh \& Shapiro, 2006).
Para ambas, a interpretação dos fatos é mais relevante do que os fatos em si. Contudo, uma diferença importante é que na meditação os conteúdos mentais não devem ser confrontados ou elaborados intencionalmente, apenas observados. Assim, a prática torna-se um aprendizado de como não se deixar influenciar pelos mesmos, os percebendo como meros fluxos mentais (Teasdale, 1999).

Outra aproximação possível entre meditação e os construtos de bem-estar descritos diz respeito à importância que estes atribuem aos diferentes domínios da vida. Uma premissa norteadora das práticas meditativas é que o exercício da atenção plena, caracterizada pela capacidade de não vincular-se automaticamente a julgamentos e elaborações, deve ser sistemático e contínuo, a fim de que se manifeste de forma permanente no funcionamento humano, em todas as suas dimensões (Austin, 1998).

Achados empíricos de pesquisas qualitativas, correlacionais e de intervenção parecem corroborar os paralelos descritos. Por exemplo, através de entrevistas foi observado que os praticantes percebem os benefícios de sua prática como abrangendo diferentes âmbitos da vida no seu cotidiano, representados por aspectos cognitivos, emocionais, sociais, físicos e espirituais (Menezes \& Dell'Aglio, 2009). Os benefícios cognitivos e emocionais estão entre os mais citados, cujo dado apóia a prerrogativa de que estes são dois aspectos centrais para a avaliação do bem-estar (Diener e cols., 2005).

Associações significativas entre meditação e bemestar também são encontradas em estudos correlacionais. Estas foram reproduzidas utilizando diferentes instrumentos de bem-estar, tal como o Questionário de Saúde Geral de Goldberg - QSG (Menezes \& Dell'Aglio, 2010) e a Escala de Bem-Estar Psicológico de Ryff - PWBS (Ortner e cols., 2007). Embora estes estudos não demonstrem causalidade, as associações encontradas têm sido respaldadas por intervenções com meditação. Nestas, o incremento do bem-estar também foi observado através de diferentes medidas, por exemplo, a Escala de Bem-Estar Psicológico - PWBS (Carmody \& Baer, 2008), a Escala de Afetos Positivo e Afeto Negativo - PANAS (Davidson e cols., 2003) e a Escala de Estados Positivos da Mente (Positive States of Mind - PSOM) (Jain e cols., 2007).

Similarmente, a redução de alguns aspectos considerados negativos ou disfuncionais também tem sido concebida como um indicador do aumento do bem- 
- estar após a prática de meditação. Entre eles, a redução de sintomas de estresse, medido pelo Inventário Breve de Sintomas (The Brief Sympton Inventory BSI) (Jain e cols., 2007) e pela Escala de Estresse Percebido (The Perceived Stress Scale) (Nyklíček \& Kuijpers, 2008; Shapiro, Oman, Thoresen, Plante, \& Flinders, 2008); de sintomas de ansiedade, medida pelo Inventário de Ansiedade de Beck (Beck Anxiety Inventory - BAI) (Chambers e cols., 2008) e pelo Inventário de Ansiedade Estado e Traço de Spielberger (Spielberger State-Trait Anxiety Inventory) (Davidson e cols., 2003); e de depressão, através do Inventário de Depressão de Beck (Beck Depression Inventory - BDI) (Chambers e cols., 2008). A diminuição destes sintomas e a sua relação com a meditação têm sido igualmente inferidas a partir da observação de níveis mais baixos de neuroticismo entre praticantes (Baer e cols., 2008; Ortner e cols., 2007).

\section{Processos Psicológicos Subjacentes}

Os dados apresentados até aqui indicam uma relação entre meditação e bem-estar. Mas por que esta associação existe e por que a meditação pode promover bem-estar? Para responder estas questões, alguns mecanismos têm sido sugeridos (Baer, 2003; Walsh \& Shapiro, 2006), sendo que neste artigo serão enfatizados três em particular: relaxamento, metacognição/mindfulness e autorregulação. Estes mecanismos são, em primeiro lugar, benefícios adquiridos com a prática. No entanto, propõe-se que eles também constituem os caminhos principais que, de forma interligada, conduzem à experiência de bem-estar.

\section{Relaxamento}

De forma ampla, o relaxamento se caracteriza por reações biológicas e emocionais contrárias às de estresse e ansiedade, as quais podem ser medidas pela menor ativação do sistema nervoso central e autônomo (Casey \& Benson, 2004). O treino de meditação parece ser uma forma de estimular estas reações. Alguns padrões característicos, que configuram um estado de relaxamento, têm sido observados em meditadores, em contraste a pessoas que foram instruídas a apenas descansar. A redução da taxa respiratória, da frequência cardíaca, da variabilidade da frequência cardíaca e da condutância da pele, assim como um padrão de atividade cerebral caracterizado por uma maior amplitude e sincronização das ondas alpha e theta nas regiões frontais estão entre os mais citados (Aftanas \& Golocheikine, 2001; Danucalov, Simões,
Kozasa, \& Leite, 2008; Takahashi e cols., 2005; Travis \& Wallace, 1999). Muitos desses padrões se correlacionam, sugerindo que o relaxamento adquirido na meditação é o resultado da ação integrada dos mesmos (Takahashi e cols., 2005).

Em geral, diferentes níveis de ativação periférica e central podem corresponder a distintas experiências e estados internos (Keil e cols., 2008), o que parece explicar porque o estado de relaxamento é normalmente acompanhado de outras respostas emocionais positivas. Na meditação, praticantes que tiveram uma condutância da pele diminuída mostraram menor suscetibilidade ao estresse induzido por filme (Goleman \& Schwartz, 1976) e aumento no autorrelato de bemestar (Ortner e cols., 2007). Em meditadores em que foi observado um aumento da sincronização de ondas alpha e theta, também houve um maior relato de experiências de bem-estar (Aftanas \& Golocheikine, 2001).

No entanto, ressalta-se que apesar de relacionadas, as técnicas de relaxamento e de meditação não são atividades idênticas e não compartilham todos os seus aspectos (Jain e cols., 2007; Lutz e cols., 2007; Ortner e cols., 2007). Algumas comparações dos efeitos da meditação com relaxamento (Ortner e cols., 2007; Takahashi e cols., 2005) e biofeedback (Cuthbert e cols., 1981) apontaram que a redução da condutância da pele, da frequência cardíaca e da taxa respiratória foi significativamente mais acentuada entre os meditadores. Também em comparação ao biofeedback, os meditadores relataram se sentir mais relaxados (Cuthbert e cols., 1981). Entende-se que a explicação para estas diferenças é o fato de a meditação incluir uma atividade mental caracterizada pela redução do processamento da informação, ou seja, o não engajamento em pensamentos associativos, discursivos e/ou ruminativos oriundos do meio interno e/ou externo, potencializando os efeitos do relaxamento, tal como a menor ativação fisiológica (Cuthbert e cols., 1981; Jain e cols., 2007).

Em suma, uma forma de esclarecer a relação entre estas técnicas é entender que o relaxamento é um dos componentes da meditação - por isso é um dos parâmetros que formam a definição operacional de meditação proposta por Cardoso e cols. (2004) - mas que o relaxamento, por si só, não é meditação. No contexto da prática meditativa, o relaxamento, através da menor ativação periférica e central, é um dos componentes que contribui para um estado emocional de bem-estar. 


\section{Metacognição/Mindfulness}

Conforme foi apresentado, a meditação mostra resultados distintos do relaxamento e esta diferença tem sido atribuída ao fato de que na meditação há um treino mental que produz uma redução do processamento da informação. Aliado a isto, este tipo de treino também tem sido associado à estimulação de funções mentais superiores, tal como metacognição e mindfulness. No presente artigo, estas serão concebidas como interdependentes, uma vez que envolvem tipos mais refinados de consciência, os quais podem contribuir para estados emocionais positivos.

A metacognição pode ser descrita como a capacidade de monitorar os próprios processos cognitivos e de usar este meta-conhecimento para regular o processamento da informação e o comportamento (Koriat, 2007). Para Teasdale (1999), no entanto, o conhecimento metacognitivo pode não ser suficiente para a mudança de padrões mentais/emocionais disfuncionais. Por isso, este autor refere-se ao insight metacognitivo (ou consciência metacognitiva). Este envolve um tipo particular de compreensão, em que pensamentos e sentimentos são percebidos como eventos mentais, e não como o próprio self. Sem desconsiderar a importância de uma pessoa conhecer/saber que seus pensamentos nem sempre são acurados (conhecimento metacognitivo), Teasdale (1999) sugere que o grande diferencial para a transformação pessoal é o insight metacognitivo, pois além de saber, é preciso sentir/experimentar os pensamentos como eventos passageiros no campo da consciência, ao invés de fatos que refletem a realidade diretamente. Em outras palavras, para que este conhecimento e estas novas representações gerem um impacto emocional, é necessário que se originem de uma aprendizagem implícita.

A prática meditativa tem sido considerada um dos caminhos para este insight, tanto na perspectiva das filosofias que a originaram, como das investigações em Psicologia (Walsh \& Shapiro, 2006). Uma das explicações é que através da meditação há um refinamento da consciência, uma vez que o treino da focalização da atenção - tanto atenção focada como monitoramento aberto - facilita o descentramento, promovendo uma desidentificação com os processos mentais, os quais são muitas vezes disfuncionais. Isto permite ao praticante manter sua atenção à experiência do momento atual, sem engajar-se em um processo de elaboração contínua ou reagir automaticamente ao processamento de qualquer estímulo. Dessa forma, a meditação possibilita que o praticante desenvolva uma percepção e uma mente menos condicionada, caracterizando um processo metacognitivo.

Já foi demonstrado que de fato os meditadores parecem ter um maior controle sobre a elaboração de pensamentos associativos em comparação a pessoas que nunca meditaram (Pagnoni, Cekic, \& Guo, 2008). Isto foi feito através da utilização de uma tarefa de decisão lexical, juntamente com o imageamento cerebral. Em determinados momentos enquanto os participantes - praticantes experientes e novatos - faziam a meditação, palavras eram apresentadas e os mesmos deviam decidir se esta era em inglês ou era uma palavra sem sentido semântico. Foi observado que a rede neural normalmente ativada na corrente de pensamentos espontâneos e no processamento conceitual regiões do córtex pré-frontal medial, córtex cingulado posterior, giro angular, giro frontal superior medial, hipocampo e giro hipocampal - teve uma ativação significativamente menor na fase pós-estímulo no grupo de meditadores experientes. Isto indica que para os novatos era mais difícil voltar ao foco da meditação e interromper a cascata de associações conceituais eliciadas pelo estímulo semântico. Já para os meditadores, o resultado apoiou a ideia de que a prática melhora a capacidade de regular, de forma voluntária, a atividade mental espontânea, ou seja, a reatividade à associação de ideias.

Alguns estudos também demonstram que a meditação auxilia na redução do processamento oriundo de estímulos internos, tal como no caso da ruminação (Chambers e cols., 2008; Shapiro e cols., 2008). Assim, com base nestes achados, sugere-se que esta capacidade de controlar o fluxo de pensamentos é uma das formas de propiciar o insight metacognitivo, visto que a mente só poderá tornar-se menos condicionada se houver uma menor reatividade ao processamento associativo. Dessa forma, podem surgir novos insights e de uma forma implícita. Apoiando esta ideia, os estudos mostram que há uma associação inversa entre os escores de ruminação e os de mindfluness, a qual tem sido descrita justamente como uma consciência menos condicionada (Chambers e cols., 2008).

Embora seja às vezes traduzida como atenção plena (Vandenberghe \& Sousa, 2006), mindfulness é uma palavra que não possui um equivalente no português, e por isso também tem sido empregada na sua forma original. Discute-se que o conceito tem raízes no $\mathrm{Bu}$ dismo e outras tradições contemplativas e, embora seja utilizado para nomear um subtipo de meditação, mindfulness é, na realidade, a própria qualidade que a prática busca cultivar (Bishop e cols., 2004). 
Esta pode ser descrita como um tipo de consciência pré-reflexiva, contrária ao funcionamento automatizado, e que resulta em uma maior clareza das experiências e do momento presente (Brown \& Ryan, 2003). Com relação à prática propriamente dita, mindfulness é definida operacionalmente a partir de dois componentes, a regulação da atenção e a abertura/aceitação do momento presente (Bishop e cols., 2004). Além disso, é importante ao menos destacar que esta qualidade também tem sido discutida fora do contexto da meditação, visto que é considerada algo inerente ao funcionamento psicológico saudável, que poderia se manifestar em qualquer pessoa (Langer, 2005).

No contexto da prática meditativa, diversos instrumentos de auto-relato têm sido desenvolvidos com o intuito de avaliar o construto, por exemplo, o Mindful Attention Awareness Scale (MAAS - Brown \& Ryan, 2003); o Freiburg Mindfulness Inventory (FMI - Walach, Buchheld, Buttenmüller, Kleinknecht, \& Schmidt, 2006); o Kentucky Inventory of Mindfulness Skills (KIMS - Baer, Smith, \& Allen, 2004); o Cognitive and Affective Mindfulness Scale_(Feldman, Hayes, Kumar, Greeson, \& Laurenceau, 2007); o Southampton Mindfulness Questionnaire (SMQ - Chadwick e cols., 2008); o Philadelphia Mindfulness Scale (Cardaciotto, Herbert, Forman, Moitra, \& Farrow, 2008); o Toronto Mindfulness Scale (TMS - Lau e cols., 2006); e o Five Facet Mindfulness Questionnaire (FFMQ - Baer e cols., 2008). Embora o objetivo deste artigo não seja uma revisão crítica de cada um destes instrumentos, é importante notar que existe uma falta de consenso entre suas estruturas, pois alguns avaliam duas ou mais facetas, enquanto outros medem mindfulness como um único construto. Há argumentos de que a utilização de diferentes facetas pode facilitar a compreensão de habilidades específicas cultivadas com a prática de meditação e como estas se relacionam com o ajustamento psicológico (Baer e cols., 2008).

Não obstante, mesmo na ausência de uma conclusão sobre a melhor solução para o construto, aqueles estudos que avaliaram a correlação dos diferentes instrumentos com a prática meditativa encontraram resultados positivos significativos (Baer e cols., 2008; Brown \& Ryan, 2003; Chadwick e cols., 2008; Lau e cols., 2006; Walach e cols., 2006). Além disso, a utilização destas medidas tem permitido corroborar a premissa das tradições contemplativas de que uma mente menos condicionada permite ao praticante cessar padrões mentais/emocionais geradores de sofrimento, alcançando níveis mais altos de bem-estar. Isto porque muitos estudos que observaram um aumento do bem-estar após a prática de meditação encontraram que mindfulness mediou este efeito (Baer e cols., 2008; Brown \& Ryan, 2003; Nyklíček \& Kuijpers, 2008; Shapiro e cols., 2008).

\section{Autorregulação}

De forma ampla, a autorregulação pode ser entendida como a habilidade de modular a cognição, a emoção e o comportamento, a fim de gerar uma melhor adaptação ao meio (Lewis \& Todd, 2007). Embora na Psicologia ainda se discuta se a autorregulação envolve apenas processos executivos e/ou processos automáticos, há consenso de que a mesma abrange o controle de respostas corticais e subcorticais. Por esta razão, é muitas vezes dividida em regulação atencional e regulação emocional, embora se argumente que a autorregulação é um processo único, envolvendo a interdependência dos diferentes níveis da atividade cerebral (Lewis \& Todd, 2007). Conforme será apresentado a seguir, diferentes medidas - neurofisiológica, imagem funcional e comportamental - podem representar processos regulatórios.

$\mathrm{Na}$ perspectiva da "ciência psicológica da meditação", esta prática tem sido descrita como autorregulatória por excelência, visto que o seu objetivo compreende exatamente aquilo que mais caracteriza a autorregulção, isto é, um maior controle dos processos internos (Lutz e cols., 2007). Diversos achados, muitos dos quais já foram discutidos ao longo deste artigo, evidenciam esta relação.

Por exemplo, iniciou-se falando da relação entre meditação e relaxamento e a influência da prática meditativa na redução da ativação autonômica. Recentemente, um estudo utilizando ressonância magnética mostrou que as regiões cerebrais implicadas na regulação autonômica parecem de fato sofrer influências com o treino da meditação (Vestergaard-Poulsen e cols., 2009). Ao comparar meditadores experientes e um grupo de pessoas que nunca havia meditado, os autores encontraram que os meditadores tinham uma maior densidade de massa cinzenta em uma região da parte inferior do tronco encefálico - o bulbo - assim como no cerebelo anterior (bilateral), no giro frontal superior e inferior esquerdo e no giro fusiforme esquerdo. A região do bulbo, a qual está diretamente relacionada a funções autonômicas, apresentou a maior diferença. Este dado indica a importância da prática regular para a neuroplasticidade e sugere que através da regulação das respostas periféricas, estas mudanças estruturais podem estar favorecendo o estado de relaxamento e o controle da reatividade neurofisiológica. 
Tendo em vista que a meditação é normalmente comparada a uma condição controle, é possível inferir que estes efeitos são gerados pelo tipo de treinamento mental característico da prática. Tang e Posner (2009) discutem que este treino gera um estado atencional particular que, uma vez aperfeiçoado, não gera fadiga e representa um trabalho integrado entre um controle executivo, considerado top-down, e um controle autonômico, bottom-up.

Uma área do cérebro que tem sido bastante implicada neste equilíbrio é o córtex cingulado anterior (CCA), cuja região parece ser significativamente mais ativada, de forma bilateral, em meditadores experientes, comparados a novatos (Holzel e cols., 2007). Este efeito foi observado especialmente na porção rostral, a qual, segundo os autores, é geralmente mais ativada quando a regulação da atenção está a serviço do processamento emocional.

Este e outros dados mostram que durante a prática de meditação é possível treinar e estimular novos arranjos cerebrais - estruturais e funcionais - assim como novos padrões de funções mentais. Estudos comportamentais ajudam a demonstrar que estes efeitos também podem ser observados fora da prática, refletindo capacidades de regulação emocional e atencional. Por exemplo, o estudo de Ortner e cols. (2007) verificou que as pessoas que aprenderam meditação durante dois meses, em comparação a pessoas que fizeram relaxamento, apresentaram um maior controle da interferência de estímulos emocionais negativos irrelevantes no processamento cognitivo - avaliar se um som concomitante à apresentação da imagem era grave ou agudo. A avaliação das figuras desagradáveis também foi menos intensa no grupo da meditação, sugerindo que este treino resulta na capacidade de interromper um processamento prolongado do estímulo negativo.

Corroborando os achados comportamentais, Ortner e cols. (2007) também observaram aumentos nos escores de bem-estar e mindfulness entre os meditadores. Portanto, parece que uma consciência menos condicionada interage com uma maior capacidade de regular o processamento de estímulos negativos, o que possivelmente resulte em experiências mais positivas.

Por fim, a atenção, a qual é considerada um componente fundamental da capacidade de se autorregular, também parece ser influenciada pela meditação. Ao combinar a tarefa comportamental do piscar atencional e a mensuração do componente $\mathrm{P} 3 \mathrm{~b}$ dos potenciais relacionados a evento, foi demonstrado que $o$ grupo que passou pelo treinamento intensivo de três meses de meditação, em comparação ao controle, apresentou um melhor desempenho na tarefa. Esta consistia em perceber um segundo estímulo acuradamente, mesmo sendo temporalmente muito próximo do primeiro (Slagter e cols., 2007). Além disso, este resultado se correlacionou com uma melhor distribuição do P3b, uma vez que a redução da amplitude do componente P3b evocado no primeiro estímulo se correlacionou com a melhor detecção do segundo estímulo no grupo da meditação. Ou seja, mesmo frente à forte competição de dois estímulos muito próximos temporalmente, o controle da alocação dos recursos para o processamento do estímulo inicial permitiu que houvesse recursos disponíveis para o processamento do estímulo posterior.

\section{CONSIDERAÇÕES FINAIS}

Segundo Huppert e cols. (2004), o estudo científico do bem-estar é especialmente importante na atualidade, pois vive-se um paradoxo. Ao mesmo tempo em que nunca houve tanta riqueza, acesso à educação, a serviços de saúde e a oportunidades de lazer, também se destaca um aumento da insatisfação de vida, altas taxas de depressão, crime e desintegração social. Para estes autores, cabe à ciência buscar formas de promover maior saúde e felicidade.

Este é justamente um dos objetivos da integração entre meditação e ciência psicológica. Com base nos achados apresentados, percebe-se que através da meditação é possível treinar a mente e desenvolver um estado de relaxamento, assim como habilidades metacognitivas e regulatórias que propiciam experiências emocionais positivas e adaptativas.

Além de levar em consideração a relevância do bem-estar para a vida em geral, o estudo científico da meditação pode auxiliar a identificar em que contextos esta prática pode ser mais eficaz para o desenvolvimento destas habilidades regulatórias. Diversas iniciativas já têm sido tomadas e esta técnica também tem tido aplicações clínicas importantes. Muitos resultados existentes decorrem de estudos com populações específicas, e programas que utilizam a meditação como principal ferramenta têm sido desenvolvidos para tratamentos complementares de transtornos psiquiátricos (Baer, 2006).

Por outro lado, ainda são necessários estudos indicando em que situações ou para que populações a meditação seria contraproducente. Além disso, um desafio para o campo é conseguir que pacientes e pes- 
soas em geral se comprometam com a prática. A meditação, assim como qualquer estratégia comportamental, requer motivação, persistência e disciplina. A motivação talvez seja a mais importante, uma vez que é fundamental para o início da prática, assim como para a sua manutenção e regularidade. A persistência é necessária, visto que, em geral, o início do treino pode ser difícil e os resultados podem não ser percebidos em curto prazo. Por fim, deveria haver disciplina, pois para que os efeitos possam realmente se manifestar e possam, por fim, caracterizar um novo padrão de funcionamento psicológico, a repetição e a regularidade são fundamentais. Embora não compreenda um mecanismo em si, sabe-se que o tempo de prática e a repetição têm um forte papel mediador do efeito da meditação sobre o bem-estar (Menezes \& Dell’Aglio, 2010).

Em suma, o aumento e a sistematização da investigação científica da prática meditativa, da sua aplicação nas áreas da saúde - em contextos clínicos e não clínicos -, assim como a publicação e divulgação destes achados podem contribuir neste sentido. No que tange ao campo da Psicologia, em especial, estes estudos podem incluir investigações qualitativas e quantitativas sobre como as pessoas vivenciam a sua prática, os seus efeitos, quais as dificuldades encontradas, o que as motiva a buscar e manter a mesma, além de outros fatores possivelmente associados, tais como traços de personalidade. A meditação também é uma alternativa para a pesquisa aplicada, através de estudos clínicos que testem sua eficácia como tratamento complementar e/ou preventivo para diversas condições psicológicas e psicossomáticas, possibilitando o estudo da tão referida relação mente-corpo. Da mesma forma, pode contribuir para a pesquisa básica, auxiliando na compreensão de processos como atenção, consciência, percepção, emoção, e suas interrelações, bem como no entendimento da plasticidade cerebral e da relação mente-cérebro. Além disso, através do estudo da meditação, a Psicologia encontra-se com outras áreas importantes e relacionadas, como Neurociências, Psiquiatria, Medicina Comportamental, Psiconeuroimunologia, entre outras. Por fim, dentro da própria ciência psicológica, a meditação pode ser estudada e aplicada dentro de diferentes abordagens, visto que todas visam ao desenvolvimento de uma mente mais saudável e a um estado de bem-estar psicológico.

No que tange o estudo da meditação no Brasil, o número de publicações indica que a produção na área ainda é restrita e recente. Dois estudos de revisão em língua portuguesa (Menezes \& Dell’Aglio, 2009;
Vandenberghe \& Sousa, 2006) buscam destacar a aplicação clínica da meditação e sua relação ao campo da psicologia. Um deles enfatiza especialmente a relação entre mindfulness - descrita tanto como prática, quanto como o tipo de consciência que se busca cultivar - e terapias cognitivo-comportamentais (Vandenberghe \& Sousa, 2006). Também existem estudos empíricos produzidos no Brasil, publicados tanto nacionalmente (por exemplo, Menezes \& Dell'Aglio, 2009), como internacionalmente (por exemplo, Menezes \& Dell'Aglio, 2010; Simões e cols., 2008). Estes investigaram aspectos discutidos neste artigo, tal como bem-estar e regulação autonômica, e concebem a meditação como uma forma de treinamento mental, mesmo quando vinculada a alguma filosofia em particular (Simões e cols., 2008). Não obstante, a produção nacional ainda é pequena e, consequentemente, ainda contribui pouco para a discussão de novos achados, tanto em nível nacional, como internacional.

\section{REFERÊNCIAS}

Obs.: Referências precedidas de um asterisco indicam estudos incluídos na revisão.

*Aftanas, L. I., \& Golocheikine, S. A. (2001). Human anterior and frontal midline theta and lower alpha reflect emotionally positive state and internalized attention: High-resolution EEG investigation of meditation. Neuroscience Letters, 310, 57-60. doi: 10.1016/S0304-3940(01)02094-8

Austin, J. H. (1998). Zen and the brain: Toward an understanding of meditation and consciousness. Cambridge: MIT Press.

*Baer, R. A. (2003). Mindfulness training as a clinical intervention: A conceptual and empirical review. Clinical Psychology: Science and Practice, 10, 125-143. doi: 10.1093/clipsy/bpg015

Baer, R. A. (Org.). (2006). Mindfulness-based treatment approaches: Clinician's guide to evidence base and applications. San Diego: Academic Press.

*Baer, R. A., Smith, G. T., \& Allen, K. B. (2004). Assessment of mindfulness by self-report: The Kentucky Inventory of Mindfulness Skills. Assessment, 11, 191-206. doi: 10.1177/1073191 104268029

*Baer, R. A., Smith, G. T., Lykins, E., Button, D., Krietemeyer, J., Sauer, S., ...Williams, J. M. G. (2008). Construct validity of the Five Facet Mindfulness Questionnaire in meditating and nonmeditating samples. Assessment, 15(3), 329-342. doi: 10.1177/ 1073191107313003

*Bishop, S. R., Lau, M., Shapiro, S., Carlson, L., Anderson, N. D., Carmody, J., ...Devins, G. (2004). Mindfulness: A proposed operational definition. Clinical Psychology: Science and Practice, 11(3), 230-241. doi:10.1093/clipsy/bph077

*Brown, K. W., \& Ryan, R. M. (2003). The benefits of being present: Mindfulness and its role in psychological well-being. Journal of Personality and Social Psychology, 84, 822-848. doi: 10.1037/0022-3514.84.4.822 
*Cardaciotto, L., Herbert, J. D., Forman, E. M., Moitra, E., \& Farrow, V. (2008). The assessment of present-moment awareness and acceptance: The Philadelphia Mindfulness Scale. Assessment, 15, 204-223. doi: 10.1177/1073191107311467

*Cardoso, R., Souza, E., Camano, L., \& Leite, J. R. (2004). Meditation in health: An operational definition. Brain Research Protocols, 14, 58-60. doi:10.1016/j.brainresprot.2004.09.002

*Carmody, J., \& Baer, R. A. (2008). Relationships between mindfulness practice and levels of mindfulness, medical and psychological symptoms and well-being in a mindfulness-based stress reduction program. Journal of Behavioral Medicine, 31, 23-33. doi: 10.1007/s10865-007-9130-7

Casey, A., \& Benson, H. (2004). Mind your heart. New York: Free Press.

*Chadwick, P., Hember, M., Symes, J., Peters, E., Kuipers, E., \& Dagnan, D. (2008). Responding mindfully to unpleasant thoughts and images: Reliability and validity of the Southampton Mindfulness Questionnaire (SMQ). British Journal of Clinical Psychology, 47, 451-455. doi:10.1348/014466508X314891

*Chambers, R., Yee Lo, B. C., \& Allen, N. B. (2008). The impact of intensive mindfulness training on attentional control, cognitive style, and affect. Cognitive Therapy and Research, 32, 303-322. doi: 10.1007/s10608-007-9119-0

*Cuthbert, B., Kristeller, J., Simons, R., Modes, R., \& Lang, P. J. (1981). Strategies of arousal control: Biofeedback, meditation, and motivation. Journal of Experimental Psychology: General, $110,518-546$.

*Danucalov, M. A. D., Simões, R. S., Kozasa, E. H., \& Leite, J. R. (2008). Cardiorespiratory and metabolic changes during yoga sessions: The effects of respiratory exercises and meditation practices. Applied Psychophysiology and Biofeedback, 33, 7781. doi: 10.1007/s10484-008-9053-2

*Davidson, R. J., Kabat-Zinn, J., Schumacher, J., Rosenkranz, M., Muller, D., Santorelli, S. F., ...Sheridan, J. F. (2003). Alterations in brain and immune function produced by mindfulness meditation. Psychosomatic Medicine, 65, 564-570. doi: 00333174/03/6504-0564

Diener, E., Lucas, R. E., \& Oishi, S. (2005). Subjective wellbeing: The science of happiness and life satisfaction. Em C. R. Snyder \& S. J. Lopez (Orgs.), Handbook of positive psychology (pp. 63-73). New York: Oxford USA Trade.

*Dimidjian, S., \& Linehan, M. M. (2003). Defining an agenda for future research on the clinical application of mindfulness practice. Clinical Psychology: Science and Practice, 10, 166-171. doi: 10.1093/clipsy/bpg019

*Feldman, G., Hayes, A., Kumar, S., Greeson, J., \& Laurenceau, J. (2007). Mindfulness and emotion regulation: The development and initial validation of the Cognitive and Affective Mindfulness Scale-Revised (CAMS-R). Journal of Psychopathology and Behavioral Assessment, 29, 177-190. doi: 10.1007/s10862-006-9035-8

Fleck, M. P. A., Louzada, S., Xavier, M., Chachamovich, E., Vieira, G., Santos, L., Pinzon, V. (1999). Aplicação da versão em português do instrumento de avaliação de qualidade de vida da Organização Mundial da Saúde (WHOQOL-100). Revista de Saúde Pública, 33, 198-205.

*Goleman, D. J., \& Schwartz, G. E. (1976). Meditation as an intervention in stress reactivity. Journal of Consulting and Clinical Psychology, 44(3), 456-466. doi: 10.1037/0022-006X. 44.3.456
*Holzel, B. K., Ott, U., Hempel, H., Hackl, A., Wolf, K., Stark, R., \& Vaitl, D. (2007). Differential engagement of anterior cingulate and adjacent medial frontal cortex in adept meditators and non-meditators. Neuroscience Letters, 421, 16-21. doi:10.1016/j.neulet.2007.04.074

Huppert, F. A., Baylis, N., \& Keverne, B. (2004). Introduction: Why do we need a science of well-being? Philosophical Transactions of the Royal Society B, 359, 1331-1332. doi:10.1098/rstb.2004.1519

*Jain, S., Shapiro, S. L., Swanick, S., Roesch, S. C., Mills, P. J., Bell, I., \& Schwartz, G. E. R. (2007). A randomized controlled trial of mindfulness meditation versus relaxation training: Effects on distress, positive states of mind, rumination and distraction. Annals of Behavioral Medicine, 33, 11-21. doi: 10.1207/s15324796abm3301_2

Keil, A., Smith, J. C., Wangelin, B. C., Sabatinelli, D., Bradley, M. M., \& Lang, P. J. (2008). Electrocortical and electrodermal responses covary as a function of emotional arousal: A singletrial analysis. Psychophysiology, 45, 516-523. doi: 10.1111/ j.1469-8986.2008.00667.x

Koriat, A. (2007). Metacognition and consciousness. Em P. D. Zelazo, M. Moscovitch, \& E. Thompson (Orgs.), The Cambridge handbook of consciousness (pp. 289-326). New York: Cambridge University Press.

Langer, E. (2005). Well-being. Em C. R. Snyder \& S. J. Lopez (Orgs.), Handbook of positive psychology (pp. 214-229). New York: Oxford USA Trade.

*Lau, M. A., Bishop, S. R., Segal, Z. V., Buis, T., Anderson, N. D., Carlson, L., \& Devins, G. (2006). The Toronto Mindfulness Scale: Development and validation. Journal of Clinical Psychology, 62, 1445-1467. doi: 10.1002/jclp.20326

Lewis, M. D., \& Todd, R. M. (2007). The self-regulating brain: Cortical-subcortical feedback and the development of intelligent action. Cognitive Development, 22, 406-430. doi: 10.1016/ j.cogdev.2007.08.004

*Lutz, A., Dunne, J., \& Davidson, R. (2007). Meditation and the neuroscience of consciousness: An introduction. Em P. D. Zelazo, M. Moscovitch, \& E. Thompson (Orgs.), The Cambridge handbook of consciousness (pp. 499-554). New York: Cambridge University Press.

*Martin, J. R. (1997). Mindfulness: A proposed common factor. Journal of Psychotherapy Integration, 7, 291-312.

*Menezes, C. B., \& Dell'Aglio, D. D. (2009). Os efeitos da meditação à luz da investigação científica em Psicologia: Revisão de literatura. Psicologia: Ciência e Profissão, 29, 276-289.

*Menezes, C. B., \& Dell'Aglio, D. D. (2009). Por que meditar? Os benefícios percebidos da prática de meditação. Psicologia em Estudo, 14, 565-573. doi: 10.1590/S1413-73722009000300018

*Menezes, C. B., \& Dell'Aglio, D. D. (2010). The relationship between the practice of sitting and silent meditation, and psychological well-being, and the effects of personality traits. Interamerican Journal of Psychology, 44, 1-10.

*Nyklíček, I., \& Kuijpers, K. F. (2008). Effects of mindfulnessbased stress reduction intervention on psychological well-being and quality of life: Is increased mindfulness indeed the mechanism? Annual Behavioral Medicine, 35, 331-340.

*Ortner, C. N. M., Kilner, S. J., \& Zelazo, P. D. (2007). Mindfulness meditation and reduced emotional interference on a cognitive task. Motivation and Emotion, 31, 271-283. doi: 10.1007/s11031-007-9076-7 
*Pagnoni, G., Cekic, M., \& Guo, Y. (2008). Thinking about notthinking: Neural correlates of conceptual processing during zen meditation. PloS ONE, 3, e3083. Retirado em 03 de fevereiro de 2010, de http://www.plosone.org, em 03/02/2010. doi: 10.1371/journal.pone.0003083

Ryff, C. D., \& Keyes, C. L. M. (1995). The structure of psychological well-being revisited. Journal of Personality and Social Psychology, 69, 719-727. doi: 10.1037/0022-3514.69.4.719

*Shapiro, S. L., Oman, D., Thoresen, C. E., Plante, T. G., \& Flinders, T. (2008). Cultivating mindfulness: Effects on well-being. Journal of Clinical Psychology, 64, 840-862. doi: 10.1002/ jclp.20491

*Slagter, H. A., Lutz, A., Greischar, L. L., Francis, A. D., Nieuwenhuis, S., Davis, J. M., Davidson, R. J. (2007). Mental training affects distribution of limited brain resources. PLoS Biology, 5. Retirado em 15 de junho de 2008, de http://www.plosbiology.org/article/info\%3Adoi\%2F10.1371\% 2Fjournal.pbio.0050138. doi:10.1371/journal.pbio.0050138

*Takahashi, T., Murata, T., Hamada, T., Omori, M., Kosaka, H., Kikushi, M., ...Wada, Y. (2005). Changes in EEG and autonomic nervous activity during meditation and their association with personality traits. International Journal of Psychophysiology, 55, 199-207. doi:10.1016/j.ijpsycho.2004.07.004

*Tang, Y., Ma, Y., Wang, J., Fan, Y., Feng, S., Lu, Q., ... Posner, M. I. (2007). Short-term meditation training improves attention and self-regulation. Proceedings of the National Academy of Sciences, 104(43), 17152-17156. doi: 10.1073_pnas.0707678104

*Tang, Y., \& Posner, M. I. (2009). Attention training and attention state training. Trends in Cognitive Sciences, 13, 222-227. doi:10.1016/j.tics.2009.01.009

*Teasdale, J. D. (1999). Metacognition, mindfulnes, and the modification of mood disorders. Clinical Psychology and Psychotherapy, 6, 146-155.
*Travis, F., \& Wallace, R. K. (1999). Autonomic and EEG patterns during eyes-closed rest and transcendental meditation (TM) practice: The basis for a neural model of TM practice. Consciousness and Cognition, 8, 302-318.

*Vandenberghe, L., \& Sousa, A. C. (2006). Mindfulness nas terapias cognitivas e comportamentais. Revista Brasileira de Terapias Cognitivas, 2, 35-44.

Veenhoven, R. (1997). Advances in understanding happiness. Revue Québécoise de Psychologie, 18, 29-74.

*Vestergaard-Poulsen, P., van Beek, M., Skewes, J., Bjarkam, C. R., Stubberup, M., Bertelsen, J., \& Roepstorff, A. (2009). Long-term meditation is associated with increased gray matter density in the brain stem. Neuroreport, 20, 170-174. doi: 10.1097/WNR.0b013e328320012a

*Walach, H., Buchheld, N., Buttenmüller, V., Kleinknecht, N., \& Schmidt, S. (2006). Measuring mindfulness - the Freiburg Mindfulness Inventory (FMI). Personality and Individual Differences, 40, 1543-1555. doi:10.1016/j.paid.2005.11.025

*Wallace, B. A., \& Shapiro, S. L. (2006). Mental balance and well-being: Building bridges between buddhism and western psychology. American Psychologist, 61, 690-701. doi: 10.1037/0003-066X.61.7.690

*Walsh, R., \& Shapiro, S. L. (2006). The meeting of meditative disciplines and western psychology: A mutually enriching dialogue. American Psychologist, 61, 227-239. doi: 10.1037/0003066X.61.3.22
Recebido: $17 / 12 / 2010$

Ultima revisão: 30/05/2011

Aceito: 06/06/2011 